\title{
Evidence for Graphene Edges Beyond Zigzag and Armchair
}

\author{
Pekka Koskinen*, ${ }^{1}$ Sami Malola, ${ }^{1}$ and Hannu Häkkinen ${ }^{1,2}$ \\ ${ }^{1}$ Department of Physics, NanoScience Center, 40014 University of Jyväskylä, Finland \\ ${ }^{2}$ Department of Chemistry, NanoScience Center, 40014 University of Jyväskylä, Finland
}

\begin{abstract}
The edges of nanoscopic objects determine most of their properties. For this reason the edges of honeycomb carbon - always considered either zigzag- or armchair-like - need special attention. In this report we provide experimental evidence confirming a previous unexpected prediction: zigzag is a metastable edge, as its planar reconstruction lowers energy and forms the most stable graphene edge. Our evidence is based on re-analyzing a recent experiment. Since the reconstructed edge, along with other unconventional edges we discuss, has distinct chemical properties, this discovery urges for care in experiments and theory - we must enter the realm beyond zigzag and armchair.
\end{abstract}

PACS numbers: 61.46.-w,64.70.Nd,61.48.De,68.37.Og

Graphene is a two-dimensional sheet of carbon atoms with the honeycomb structure[1, and it underlies other carbon allotropes like graphite, carbon nanotubes, and fullerenes 2. If you cut honeycomb carbon in a random orientation, the edges become purely zigzag- or armchairlike, or an alternating series of zigzag and armchair segments. So far these edges have been taken for granted, both in experiment and in theory.

The edges of graphene are prominent for several reasons. For example, carbon nanotubes are made from curved graphene sheets, and the edges determine how they grow 3, 4] or make contacts [5]. Furthermore, conductive 6], mechanical and elastic properties [7, 8, 9, as well as chemical properties 10 of graphene nanoribbons depend crucially on the edge properties (edge profile reflects the symmetry inside ribbons). One example is the electronic edge state at the zigzag edge, completely missing from the armchair edge 11].

In a recent theoretical work Koskinen et al. considered also other than pure zigzag or armchair edges[12]. Computer simulations using density-functional theory predicted, among other results, the existence of a reconstructed zigzag edge, shown in Fig. 11. The energy of the edge is lowered by $0.35 \mathrm{eV} / \AA(1.7 \mathrm{eV}$ per two adjacent hexagons) when zigzag reconstructs; this makes the reconstructed zigzag the most stable graphene edge. These results have later been confirmed in subsequent theoretical work 9 , 13, 14. Furthermore, besides lowering the energy, the reconstruction changes the edges' chemical properties. The strong dangling bonds, responsible for the reactivity of the zigzag edge, are removed by the reconstruction due to the formation of triple-bonds in the nearly linear armrest parts 15 (cf. Fig. 17). Up to now this edge has been an academic curiosity, but here we present experimental evidence confirming the prediction: zigzag and armchair edges do have company.

\footnotetext{
*Author to whom correspondence should be addressed.
}
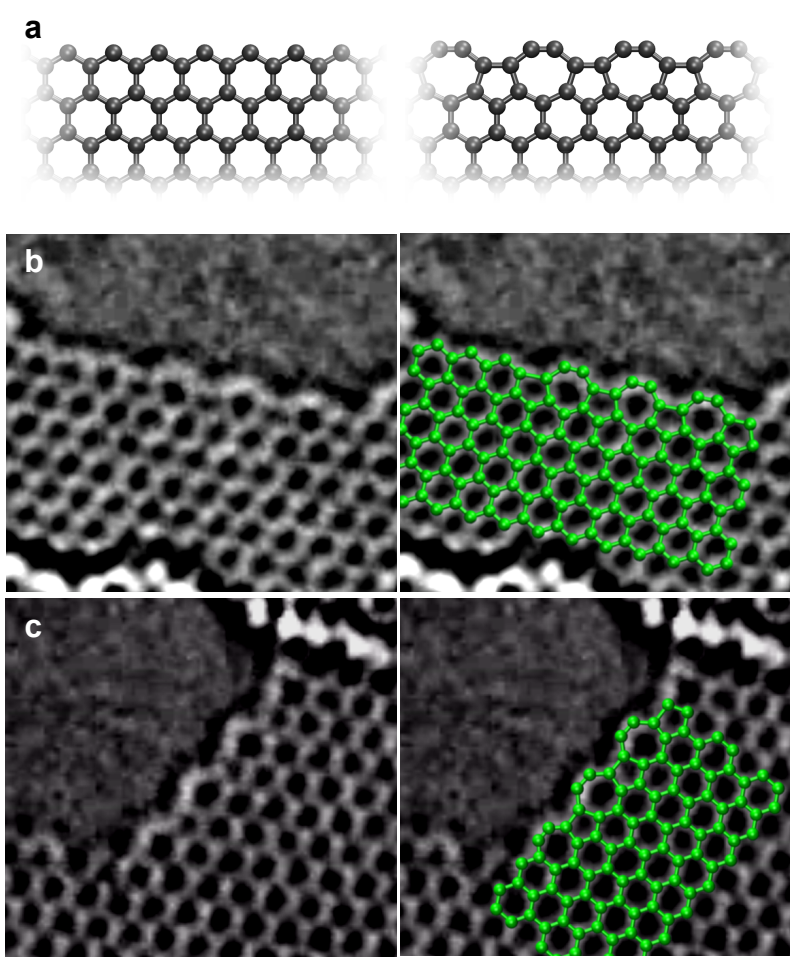

FIG. 1: (color online) Experimental evidence for zigzag reconstruction. (a) Normal zigzag edge (left) and the reconstructed zigzag edge (right), as predicted in Ref. 12 (b) Frame number 103 from the Movie S1 of Ref. 16, showing the zigzag edge reconstruction (left), with a highlighted structure assignment (right). Note the transition from pure zigzag to reconstructed zigzag edge. (c) Frame number 64 from the same movie with another reconstructed zigzag edge (left), and a structure assignment (right). Printed with the kind permission of authors of Ref. 16.

A recent impressive experiment by Girit et al. ("the experiment" from now on) imaged the edges of a freestanding graphene by aberration-corrected transmission electron microscopy (TEM) 16]. Sub-Ångström resolu- 
tion enabled the analysis of edge structures and dynamics with atom precision. The movement of atoms at the edge, driven by collisions with accelerated electrons, could be understood by simple arguments. The experimental report was supplemented by a movie (Movie S1) that animated the edge dynamics with 110 frames corresponding to some 10 minutes of real time (1 second exposure for each imaged frame with 4 second breaks). Figs. 1 $1 \mathrm{p}$ and 1. show two frames where the reconstructed zigzag edges are apparent (such frames were easy to find). The analysis in the original experimental report considered only zigzag and armchair edges because they contain regular hexagons; other polygons were rejected since they gave merely a messy appearance for the edges. This is natural, because - until now - no other edge structures have been recognized in an experiment; any deviations have been viewed as local defects. But since now Fig. 19 gives a fresh viewpoint for the experiment, it becomes evident that the reconstructed zigzag edge is a well-defined, stable, and proper edge structure in itself. On the account of this experimental discovery, we term the reconstructed zigzag edge the reczag edge. In the experiment reczag edges appear with fair abundance, and they sometimes persist for well more than ten seconds in real-time (several frames).

But how can we tell that zigzag really is metastable? First of all, the energy difference between zigzag and reczag edges is large enough to be theoretically beyond any doubts - from that standpoint the reczag edge has been nothing but waiting for discovery 12 . But also experimental considerations support metastability. As carefully explained in the experimental article, zigzag edges appear more abundant than armchair edges due to dynamic and kinematic effects related to TEM imaging, not due to lower energy[16]. In short, when TEMaccelerated electrons cause damage and kick doubly coordinated atoms at the edges, the zigzag edges are repaired rapidly by diffusing single carbon atoms, while for armchair the repair requires two atoms, hence it is slower. The reczag edge has an edge profile similar to armchair, and is therefore also discriminated by the TEM imaging process. Furthermore, in the experiment the edge is eroded so that graphene with pristine hexagons is revealed during the course of time, favoring zigzag instead of reczag edge (that contains other polygons). Regardless of these discriminating factors, fairly long segments of the reczag edges can be observed.

A systematic inspection of the experimental data reveals short segments of the other predicted edges as well, such as an armchair edge with pentagons in Fig. 2 a, or an armchair edge with two adjacent heptagons in Fig. 2b [12]. In fact, Ref. 9 predicted a partial metastability also for the armchair edge: it can relieve edge stress by forming heptagons at the edge and hereby decrease energy, attaining energy minimum with $\sim 30 \%$ heptagon concentration. However, since there are no long segments

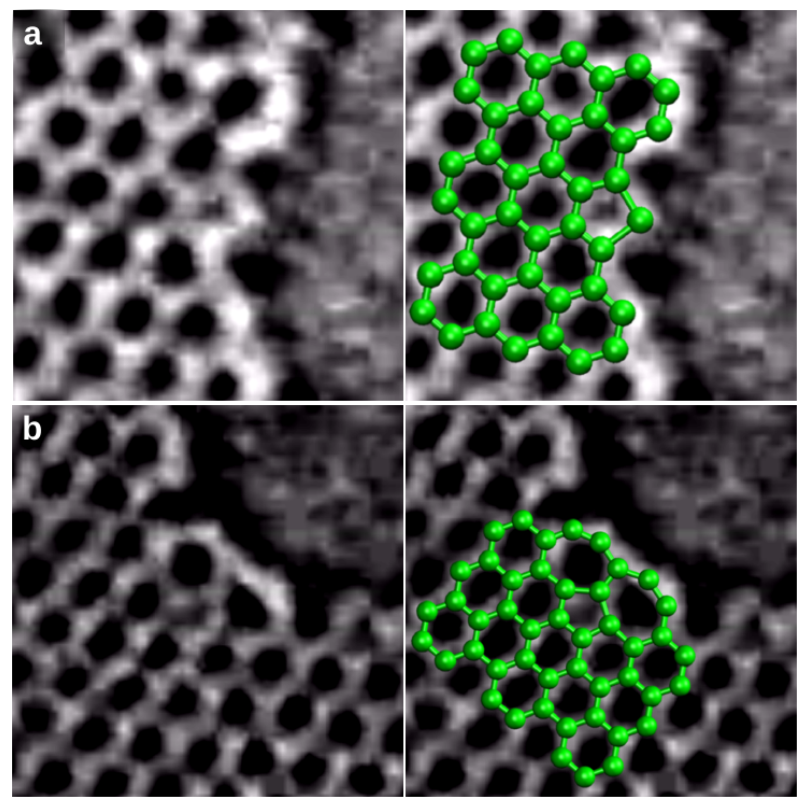

FIG. 2: (color online) Segments of other graphene edges, as seen from frame details of the Movie S1 of Ref. 16. (a) Detail of frame 73 (left) with structure assignment (right), revealing an armchair edge with pentagons. (b) Detail of frame 106 (left) with structure assignment (right), revealing an armchair edge with hexagons and two heptagons. Printed with the kind permission of authors of Ref. 16.

of armchair edges in the experiment, the edge stress does not need any relief, and heptagons at the armchair edge are only rarely observed (the energy difference is small, too).

An interesting experimental detail is the brightness in the armrest parts in armchair and reczag edges. It is due to the larger electron density between the armrest atoms, in chemical triple bonds - observed directly by TEM[15].

This Brief Report has a brief message: the reczag edge really exists - and makes a difference. In current frantic graphene research this is important to digest. First, less edge stress but more mechanical stiffness induces different warping and curling properties for reczag. [8, 9, 17] Second, reczag has vibrational properties akin to those of armchair; this is a dangerous origin for wrong identification [8, 18. Third, while zigzag's the so-called edge-state survives the reconstruction (because its origin is bulk, not edge locally), the dangling bond band in reczag moves away from the Fermi-level, trembling edge's conductive properties. 12] Fourth, and most important, reczag is chemically less reactive than zigzag. 12] A hydrogen-passivated zigzag edge, on the other hand, is more stable than hydrogen-passivated reczag edge. [12, 13 ]

Note also that, unfortunately, graphene edges cannot be identified from the bulk lattice orientation anymore.

The observations in this report are trivial, but they have solid significance. Several properties of reczag, as 
listed above, are different from the properties of zigzag, yielding different interactions and signatures at the edge. Hence the reczag edge, now with an experimental verification, has to be considered - even re-considered - in any theoretical or experimental work involving honeycomb carbon.

Note added in proof: Another experiment (Chuvilin et al., arXiv:0905.3090) has also confirmed our observations.

We acknowledge the Academy of Finland for funding and the authors of Ref. 16 for their kind permission to print their data. Figures reprinted with permission from AAAS.

† Electronic address: pekka.koskinen@iki.fi

[1] K. S. Novoselov, A. K. Geim, S. V. Morozov, D. Jiang, Y. Zhang, S. V. Dubonos, I. V. Grigorieva, and A. A. Firsov, Science 306, 666 (2004).

[2] A. K. Geim and K. S. Novoselov, Nature materials 6, 183 (2007).

[3] Y. H. Lee, S. G. Kim, and D. Tomanek, Phys. Rev. Lett. 78, 2393 (1997).

[4] E. Loginova, N. C. Bartelt, P. J. Feibelman, and K. F. McCarty, New J. Phys. 10, 093026 (2008).

[5] K. Jian, A. Yan, I. Kulaots, G. P. Crawford, and R. Hurt, Carbon 44, 2102 (2006).
[6] X. Jia, M. Hofmann, V. Meunier, B. G. Sumpter, J. Campos-Delgado, J. M. Romo-Herrera, H. Son, Y.P. Hsieh, A. Reina, J. Kong, et al., Science 323, 1701 (2009).

[7] S. Jun, Phys. Rev. B 78, 073405 (2008).

[8] S. Malola, H. Häkkinen, and P. Koskinen, Eur. Phys. J. D 52, 71 (2009).

[9] B. Huang, M. Liu, N. Su, J. Wu, W. Duan, B. Gu, and F. Liu, Phys. Rev. Lett. 102, 166404 (2009).

[10] D. Jiang, B. G. Sumpter, and S. Dai, J. Chem. Phys. 126, 134701 (2007).

[11] Y. Kobayashi, K.-I. Fukui, T. Enoki, and K. Kusakabe, Phys. Rev. B 73, 125415 (2006).

[12] P. Koskinen, S. Malola, and H. Häkkinen, Phys. Rev. Lett. 101, 115502 (2008).

[13] T. Wassmann, A. P. Seitsonen, A. M. Saitta, M. Lazzeri, and F. Mauri, Phys. Rev. Lett. 101, 096402 (2008).

[14] C. D. Reddy, A. Ramasubramaniam, V. B. Shenoy, and W.-W. Zhang, Appl. Phys. Lett. 94, 101904 (2009).

[15] T. Kawai, Y. Miyamoto, O. Sugino, and Y. Koga, Phys. Rev. B 62, R16349 (2000).

[16] Ç. Ö. Girit, J. C. Meyer, R. Erni, M. D. Rossell, C. Kisielowski, L. Yang, C.-H. Park, M. F. Crommie, M. L. Cohen, S. G. Louie, et al., Science 323, 1705 (2009).

[17] V. B. Shenoy, C. D. Reddy, A. Ramasubramaniam, and Y. W. Zhang, Phys. Rev. Lett. 101, 245501 (2008).

[18] J. H. Lan, J.-S. Wand, C. K. Gan, and S. K. Chin, Phys. Rev. B 79, 115401 (2009). 